\title{
An Artificial Neural Network for the Prediction of Penetration Height of Aerated Elliptical Liquid Jets in Gaseous Crossflows
}

\author{
Mehdi Jadidi ${ }^{1}$, Sana Shaghaghian ${ }^{2}$, Seth B. Dworkin ${ }^{1}$, Ali Dolatabadi*2 \\ ${ }^{1}$ Department of Mechanical and Industrial Engineering, Ryerson University, Toronto, Canada \\ ${ }^{2}$ Department of Mechanical and Industrial Engineering, University of Toronto, Ontario, \\ Canada \\ *Corresponding author email: dolat@mie.utoronto.ca
}

\begin{abstract}
The breakup of a liquid jet by a high-velocity gaseous crossflow has many applications in industry. Penetration height of the liquid jets in crossflows is considered as the main parameter of interest, and several empirical correlations for it have been developed by many researchers. However, recent studies show that significant differences between the predictions of the available correlations exist since the liquid jet in crossflow is a complex process and the penetration height depends on many parameters and variables. In the present study, it is shown that, although developing an accurate explicit equation or model is difficult, an Artificial Neural Network (ANN) is able to estimate the penetration height precisely. To train and test the network, input and output data have been obtained from experiments conducted in a wind tunnel. Overall, 48 different experiments have been performed, and 45 cases have been selected and partitioned into two parts: $80 \%$ for training and $20 \%$ for testing. Afterward, the remaining three cases have been used independently to test the network performance rigorously. In summary, it is revealed that ANNs enable the accurate prediction of penetration heights and have great potential to be used for more complicated operating conditions.
\end{abstract}

\section{Keywords}

Liquid jet in crossflow, Penetration height, Artificial neural network, Aerated liquid, Elliptic jet

\section{Introduction}

Liquid jet atomization in a subsonic gaseous crossflow (LJIC) is an important phenomenon happening in gas turbines, augmentors, and thermal sprays [1]-[3]. It is an extremely complicated process that has been widely investigated experimentally over the past years. The focus of most experimental works is on the penetration height of a liquid jet in a crossflow since it mainly controls the efficiency and performance in the mentioned systems [4]. Indeed, although several parameters like column and surface breakup, droplet size and velocity distributions, Sauter mean diameter (SMD), etc. have been used to study the LJIC comprehensively, the penetration height has been considered as one of the most important parameters since it indicates the location of droplets in the field and shows how well the liquid is mixed with the gas flow [4], [5]. It is worth mentioning that, in the application of thermal spray coatings, the location of droplets in the field is extremely important as low or high penetration height will significantly reduce the deposition rate [6].

So far, different experiments have been conducted under various operating conditions and several empirical correlations have been developed to estimate the liquid penetration height in crossflows [4]. In most of these empirical correlations, the penetration height is simply related to liquid-to-gas momentum flux ratio and normalized distance in the crossflow direction. For more complicated cases like liquid jets in high-pressure high-temperature crossflows, other nondimensional numbers such as gaseous Reynolds and Weber numbers are also included [4]. 
Recently, No [4] compared the predictions of about 40 different existing correlations and found clear discrepancies between the results. The main reason relies on the fact that the penetration height is a function of various parameters and variables like crossflow and liquid Reynolds numbers, Weber number, liquid-to-gas density ratio, temperature ratio (e.g. the dependency of surface tension, viscosity and density on temperature), nozzle geometry (e.g. round-edge or sharp-edge) and its location, the turbulent intensity of liquid jets and crossflows, orifice shape (circular, elliptical, rectangular orifices etc.), the presence of aerating gas within the liquid, and etc. [2], [7]-[10]. In addition, many researchers attribute the stated discrepancies to the measurement techniques incorporated to derive the correlations as well as the challenges in finding the boundaries of the sprays [4].

In many applications, more parameters and variables are involved so that estimating the penetration height becomes significantly more challenging. For example, in suspension plasma spray, the liquids are typically non-Newtonian, and the surface tension and viscosity are time-dependent [11]. In addition, the crossflow characteristics like velocity, temperature, density, viscosity, etc. depend on both space and time [12]. Moreover, due to hydrodynamic instabilities, a pulsed suspension liquid jet usually interacts with the crossflow [3], [5].

The above discussion makes it clear that developing an accurate explicit equation or model, which considers the effects of several parameters for prediction of the penetration height is challenging. However, machine learning and deep learning approaches such as Artificial Neural Networks (ANNs), which have attracted much attention recently, have been successfully applied for various difficult multi-input multi-output nonlinear regression problems [13]. In the current study, the capability of ANNs for prediction of the liquid penetration height under complicated operating conditions is examined. First, to gather validated data, aerated elliptical and circular liquid jets in gaseous crossflows have been studied experimentally in a wind tunnel using a high-speed camera and shadowgraph technique. In these experiments, the nozzle ellipticity, orientation, gas-to-liquid mass flow rate ratio $(G L R)$, and $q$ (which is defined as the liquid-to-crossflow momentum flux ratio at $G L R=0$ ) have been changed and their effects on the penetration height have been obtained. Then, a neural network is developed to relate the penetration height to the mentioned nondimensional numbers as well as normalized distance in the jet direction. In the following sections, the experimental methodology, fundamentals of ANN, and the results are discussed.

\section{Experimental Methodology}

An open-loop subsonic wind tunnel is used here to perform the experiments. The test section, which is made of clear acrylic, has a cross section of $100 \times 100 \mathrm{~mm}^{2}$ and a length of $750 \mathrm{~mm}$. The PIV result has shown that the air velocity inside the test section is uniform (except for the thin boundary layers on the walls) and can reach $45 \mathrm{~m} / \mathrm{s}$ [14]. In the present study, the air velocity inside the test section is measured by a Pitot tube.

The liquid injection system includes a pressure tank for distilled water, an effervescent atomizer, air supply, two regulators (one for controlling the water pressure inside the tank and another for supplying the effervescent nozzle with the aerating gas), and two rotameters to control water and aeration gas flowrates. A schematic of the test setup is shown in Figure 1. The atomizer is designed based on the outside-in gas injection concept [15]. In this respect, the water flows inside a perforated tube and the air is injected into it through 24 holes. The diameter of each hole is $1 \mathrm{~mm}$. Furthermore, to avoid any undesirable disturbances, the nozzle exit is set flush with the inner surface of the test section. In addition, two orifices are designed as the bottom part of the atomizer: 1 . a circular orifice with a diameter of $0.94 \mathrm{~mm}$ and 2. an elliptical orifice with the major and minor axes of 2.03 and $0.67 \mathrm{~mm}$, respectively. As discussed 
in our previous paper [14], for elliptical jets in crossflows, the aspect ratio $\left(A_{r}\right)$ is typically defined as the ratio of the axis perpendicular to the crossflow direction, to the axis parallel to the crossflow direction (see Figure 2). In this case, both nozzles' shapes and orientations can be taken into account. In this study, the elliptical orifice has aspect ratios of 3 and 0.3 , and the circular orifice has an aspect ratio of 1.

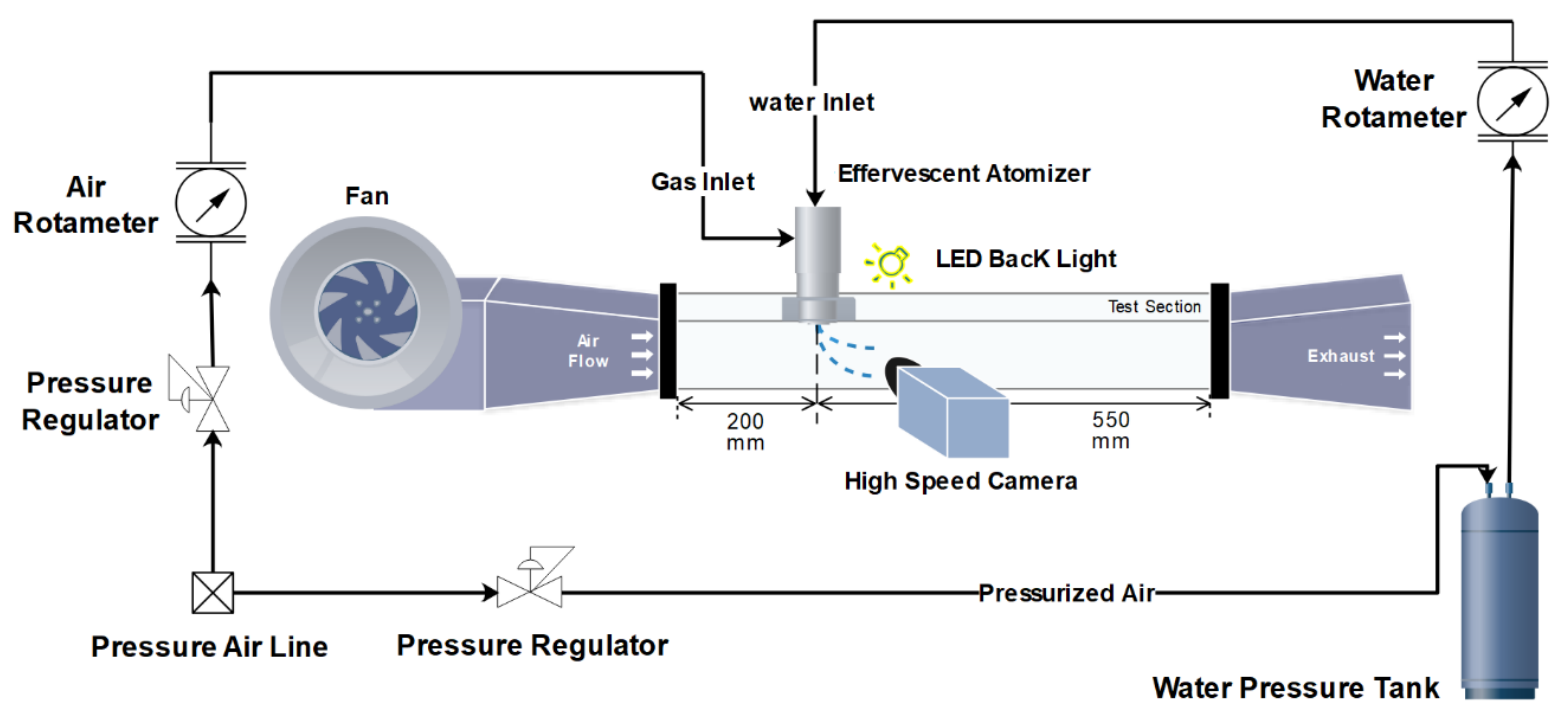

Figure 1. Schematic of the wind tunnel, spray injection, and high-speed imaging systems.

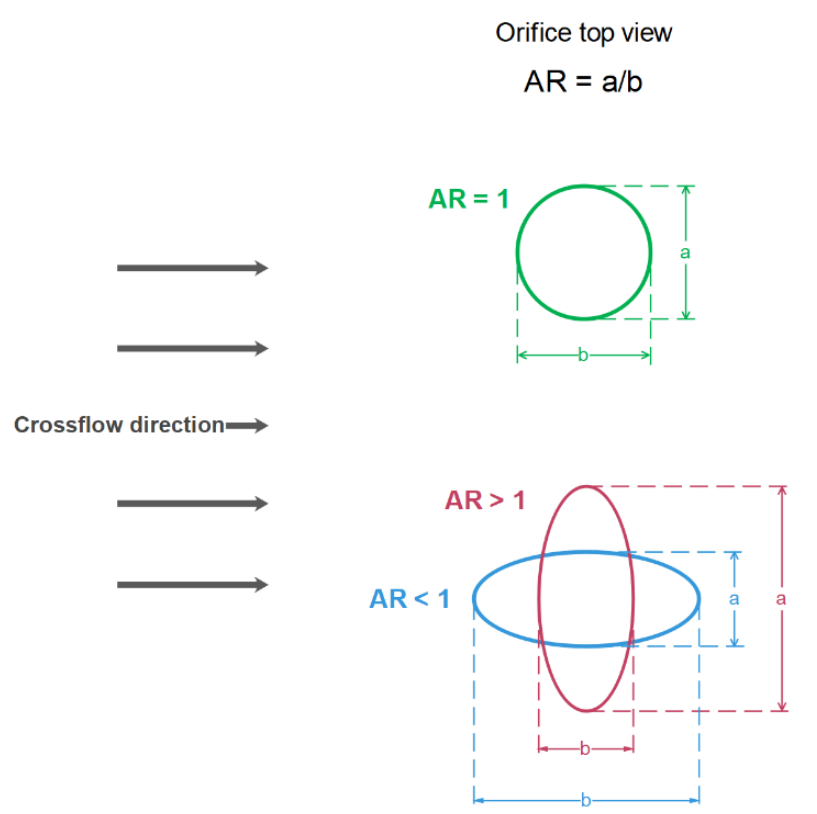

Figure 2. Schematic of aspect ratios of the elliptical and circular orifices.

In addition to $A_{r}$, other nondimensional numbers such as $q$ and $G L R$ have significant influences on the penetration height. To alter $q$, the wind tunnel velocity or the liquid flow rate is changed. However, GLR is varied by changing the aerating gas flow rate. In this study, $q$ and $G L R$ are between 2-23 and 0-7\%, respectively, and, overall, 48 different tests have been conducted. A high-speed camera (Photron SA1.1) with a Nikon lens (AF Micro Nikkor 105mm 1:2.8) has been used to record the trajectory and breakup of liquid jets in the crossflow. The camera resolution, shutter speed, and framerates have been set to $1024 \times 1024$ pixels, $1 \mu$ s, and 5000 
frames per second, respectively. Furthermore, an LED (150 W, GS Vitec Multiled QT) has been installed behind the test section as the backlight source.

After running the tests and saving the side-view images of the sprays with a personal computer connected to the camera, the ImageJ software (which is an open source image processing program) is used to detect the penetration height of the liquid jets and to gather the input and output datasets. A procedure that is very common in the field of LJIC is applied [16], [17]. That is, for each test, at first, the 5000 raw images are superimposed to obtain an averaged image. Then, the background (i.e. the image taken before injecting the liquid) is subtracted from that averaged image. Afterward, a threshold of $90 \%$ is applied to the resultant image to reduce the noise. Finally, a series of points is placed manually along the windward trajectory to obtain the penetration height. More information about this approach can be found in [16], [17]. In Figure 3 , an image taken by the high speed camera as well as the curve of penetration height are shown for one case as an example.

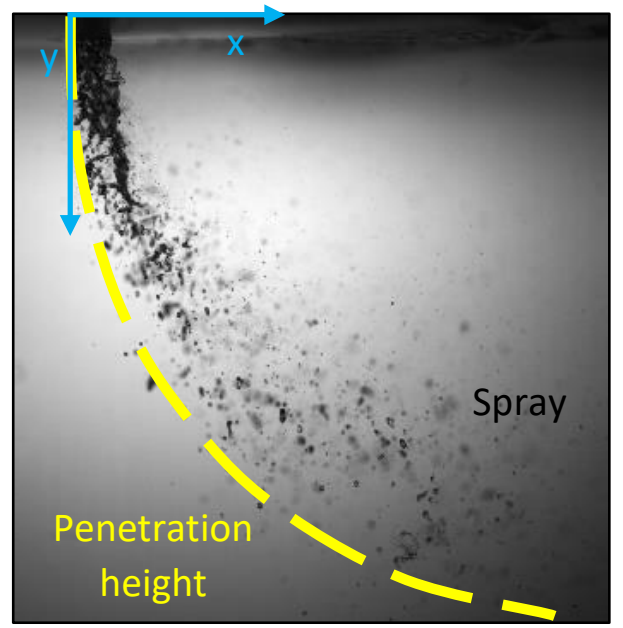

Figure 3. A sample of shadowgraph image and the obtained penetration height ( $G L R=7 \%, q=4$, and $\left.A_{r}=0.3\right)$.

\section{Artificial Neural Network (ANN)}

As represented in Figure 4, every ANN has at least three sections: the input, hidden layer(s), and output. Each section is usually referred to as a layer. In general, each network has one input, one output and can have multiple hidden layers depending on the desired accuracy and available computational power [13], [18]. In each layer, several nodes, or neurons (shown as circles in Figure 4) are included. The number of nodes in the input and output layers is equal to the number of input and output variables defined by the user. However, the number of nodes in the hidden layers is flexible. As shown, any node in the input layer is linked to every single node in the first hidden layer, and similarly, any node in the first hidden layer is connected to every single node in the second hidden layer, and so on, until the output layer is reached. A weight is associated with each connection between the two nodes. For a given node, the output is calculated by applying an activation function (such as hyperbolic tangent, identity, and rectified linear unit (relu)) to the weighted sum of inputs, and a bias. At first, these weights and biases are random, however, they are tuned by algorithms such as gradient descent and backpropagation [13]. For more information about ANN, weights and biases, optimization algorithms, etc. the interested readers are referred to several books and articles published in this area such as [13].

In the current work, the concept of supervised learning is applied. In this regard, 45 experimental tests are chosen and split into two parts: $80 \%$ for training and $20 \%$ for testing. 
The network can see and learn from the training data and the weights and biases can be adjusted based on the error. However, the testing data, which includes unseen data (not used for training), is used to analyze the network performance and generalization. In order to perform further tests, the remaining three cases are fed independently to the network and the outputs are compared with the experimental results. The details of these three cases are shown in Table 1.

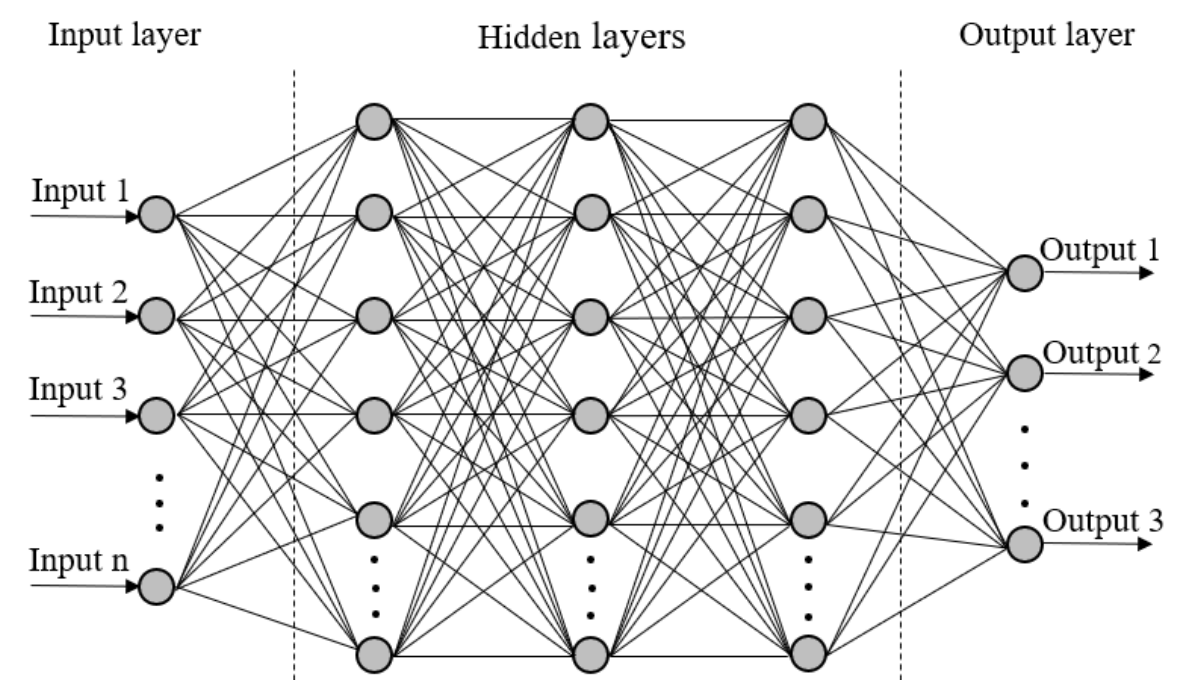

Figure 4. A schematic representation of an artificial neural network [18].

Table 1 - The three cases used for testing the network

\begin{tabular}{c|ccc}
\hline & $q$ & $A_{r}$ & GLR \\
\hline Set 1 & 4 & 0.3 & 7 \\
Set 2 & 23 & 3 & 0 \\
Set 3 & 8 & 1 & 4.5
\end{tabular}

In the present study, the input variables are $y / d, q, G L R$, and $A_{r}$, while the output parameter is $x / d$. In the field of LJIC, $x$ and $y$ are typically in the crossflow and liquid jet directions, respectively (see Figure 3 ). These parameters are normalized by the equivalent diameter of the orifices, $d$. For the circular and the elliptical orifices, the equivalent diameter is 0.94 and $1.16 \mathrm{~mm}$, respectively. We have chosen the parameter $y / d$ as the input since it is always nonnegative and results in better performance and lower errors. It should be pointed out that, $x / d$ can be negative depending on $q, G L R$, and $A_{r}$ (see the result section).

Standardization is used in the present study to rescale the datasets so that they have a mean value of 0 and a standard deviation of 1 . This technique is generally advantageous when the data has varying scales. In addition, grid searching is applied for optimizing hyperparameters and selecting the network with the best performance. The network performance was evaluated using mean squared error (MSE), R-squared value, and explained variance score. A full list of the hyperparameters as well as their values tested in our grid search is shown in Table 2. Here, the learning rate determines at which pace the weights and biases get updated. It can be fixed or adaptively varied during training to improve the computational time and numerical convergence. An epoch refers to one iteration where the model sees the entire training dataset to update the weights and biases. Moreover, the regularization parameter is applied to ensure the model is not overfitting the training dataset [13]. 
Table 2 - Hyperparameters and their tested values in the grid search

\begin{tabular}{c|c}
\hline No. of hidden layers & $1,2,3$ \\
\hline No. of nodes in each hidden layer & $10,20,30, \ldots, 180,190,200$ \\
\hline Learning rate & constant and adaptive \\
\hline Initial learning rate & $0.0005,0.0008,0.001,0.003,0.005,0.008,0.01,0.1,0.5$ \\
& $50,75,100,125,150,175,200,300,500$ \\
\hline No. of epochs & identity, tanh, relu \\
\hline Activation functions & stochastic gradient descent (sgd), adam \\
\hline Solvers & $0.0001,0.0005,0.0008,0.001,0.005,0.008,0.01$, \\
Alpha (L2 regularization & $0.05,0.08,0.1,0.5$ \\
parameter) &
\end{tabular}

\section{Results}

In Figure 5, some plots are provided to reveal the dependency of liquid penetration height on $G L R, q$, and $A_{r}$. As can be seen, by increasing $G L R$ and $q$, the penetration height increases. However, when $q$ and $G L R$ are fixed, the penetration height of the circular nozzle $\left(A_{r}=1\right)$ is the highest. In addition, as mentioned, for some cases, $x / d$ can be negative values. It should be noted that not all of the experimental data is presented here because discussing the results would be unnecessarily lengthy and beyond the scope of the current paper. In subsequent work, all the experimental results will be presented and discussed.
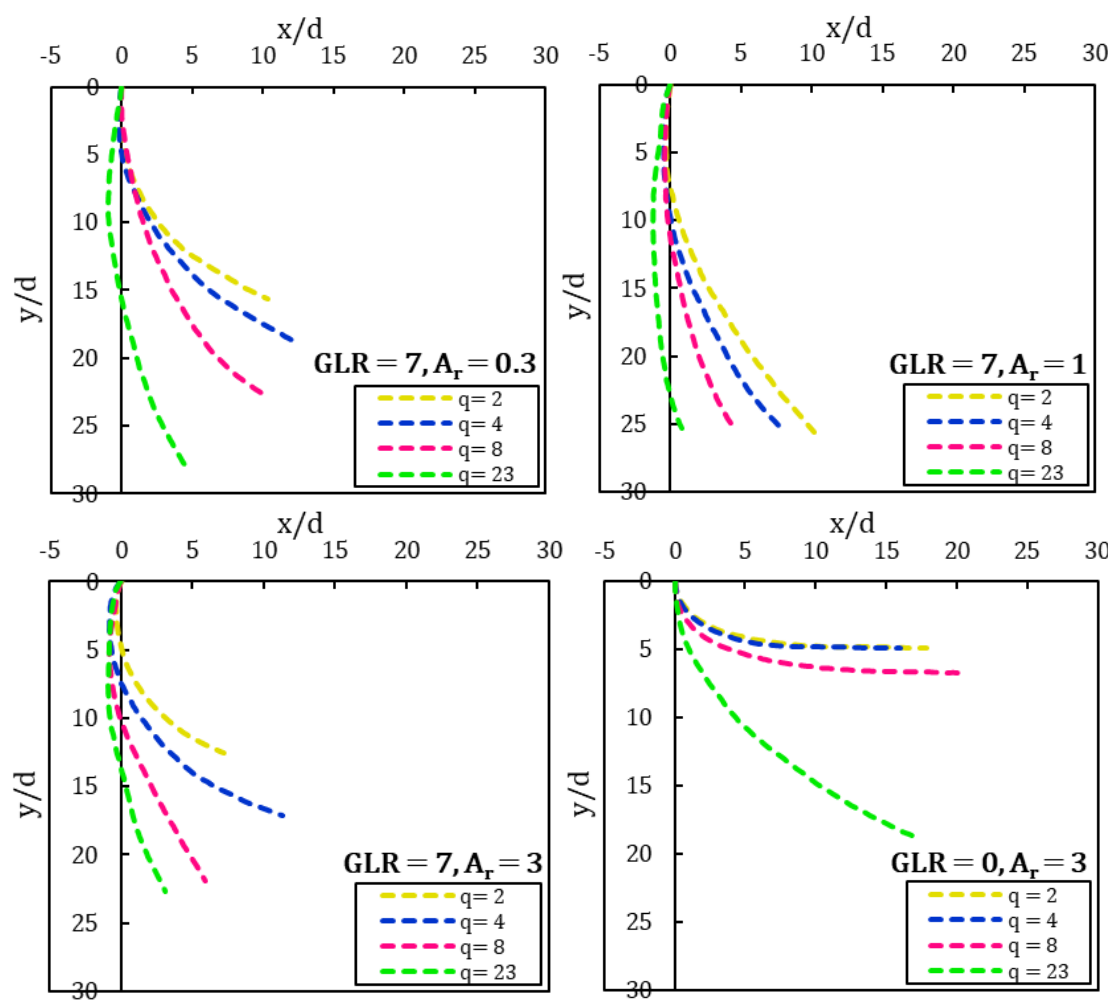

Figure 5. Liquid penetration heights obtained from the experiments.

The network with the best performance had three layers including 140 nodes in the first layer, 130 nodes in the second layer, and 130 nodes in the third layer. The solver, activation function, and learning rate were stochastic gradient descent, rectified linear unit (relu), and adaptive, respectively. The number of iterations (epochs) was 100 , and alpha and the initial learning rate were both 0.008 . In Figure 6, the predicted values for the testing dataset (i.e. the $20 \%$ 
test data stated above) are compared with the actual values obtained from the experiments. Here, the MSE, R-squared value, explained variance score, and the maximum absolute error are $0.146,0.997,0.997$, and 3.186 , respectively.

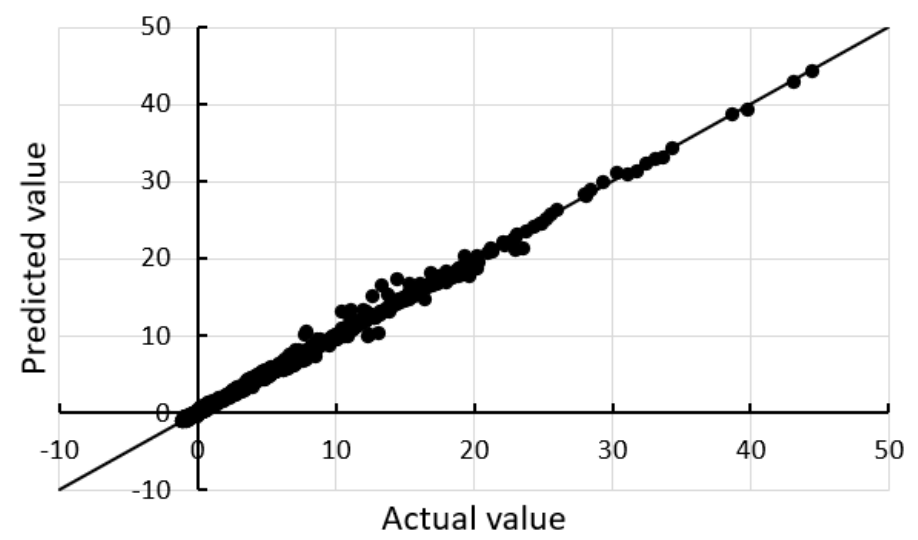

Figure 6. Predicted values vs. actual values (from the experiment) for the testing dataset.

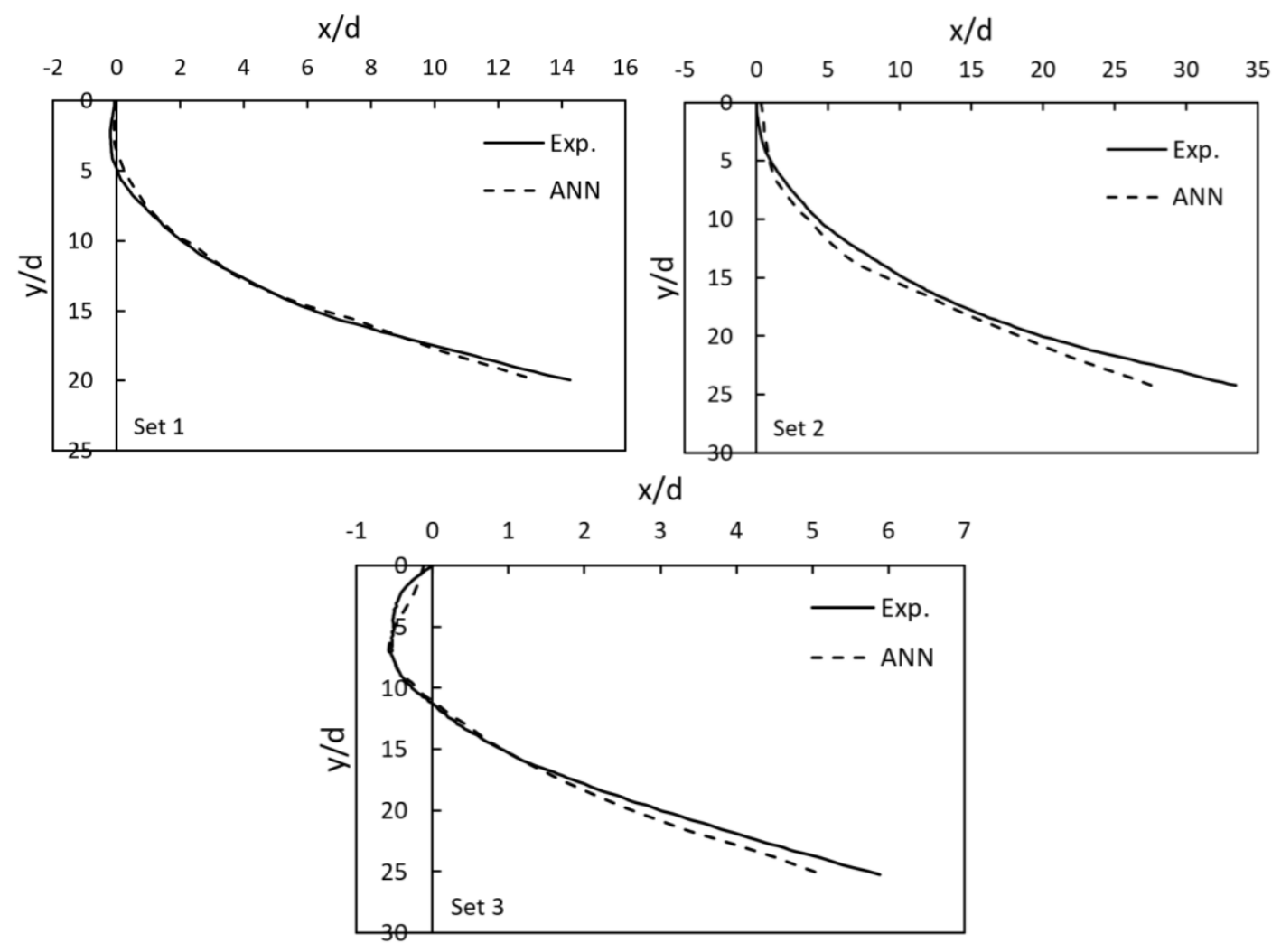

Figure 7. Network's predictions for sets 1-3 versus the experimental data.

Table 3 - MSE, R-squared value, and explained variance score for sets 1-3

\begin{tabular}{c|ccc}
\hline & MSE & R-squared & Explained variance score \\
\hline Set 1 & 0.095 & 0.994 & 0.994 \\
Set 2 & 4.386 & 0.94 & 0.969 \\
Set 3 & 0.056 & 0.977 & 0.98
\end{tabular}


In Figure 7, the predicted values for test cases, referred to as sets 1-3 (see Table 1) are compared with their corresponding experimental data. Moreover, Table 3 shows the MSE, Rsquared value as well as explained variance score for these cases. As can be seen, the network is able to predict the trends and the values of liquid penetration heights accurately.

\section{Conclusions}

In the present study, an Artificial Neural Network (ANN) has been used to predict the penetration height of a liquid jet in a gaseous crossflow. Experimental studies have been performed in a wind tunnel to gather the input/output data to train and test the network. In these experiments, elliptical and circular nozzles have been utilized and a wide range of gasto-liquid mass flow rate ratio and liquid-to-gas momentum flux ratio has been considered. After training the network, different tests have been done to analyze the network performance. Overall, the prediction heights have been accurately estimated by the network, which demonstrates the great potential of ANNs to be used for more complex conditions.

\section{Acknowledgments}

The authors acknowledge the Canadian Research Chairs program and the Natural Sciences and Engineering Research Council of Canada (NSERC) for their financial support.

\section{References}

[1] Broumand, M., and Birouk, M., 2016, Progress in Energy and Combustion Science, 57, pp. $1-29$.

[2] Herrmann, M., 2011, Proceedings of the Combustion Institute, 33(2), pp. 2079-2088.

[3] Jadidi, M., Moghtadernejad, S., Dolatabadi, A., 2016, Materials and Design, 110, pp. 425435.

[4] No, S.Y., 2015, International Journal of Spray and Combustion Dynamics, 7(4), pp. 283314.

[5] Jadidi, M., Vardelle, A., Dolatabadi, A., Moreau, C., 2018, "Heat Transfer in Suspension Plasma Spraying." in Handbook of Thermal Science and Engineering, Springer International Publishing, pp. 2923-2966.

[6] Jabbari, F., Jadidi, M., Wuthrich, R., Dolatabadi, A., 2014, Journal of Thermal Spray Technology, 23(1-2), pp. 3-13.

[7] Broumand, M., Rigby, G., Birouk, M., 2017, Flow, Turbulence and Combustion, 99(1), pp. 153-171.

[8] Broumand, M., Birouk, M., Mahmoodi, S.V., 2019, Journal of Fluid Mechanics, 879, pp. 775-792.

[9] Li, X., and Soteriou, M.C., 2018, International Journal of Multiphase Flow, 104, pp. 214232.

[10] Li, X., Gao, H., Soteriou, M.C., 2017, Physics of Fluids, 29, 082103.

[11] Jadidi, M., Moghtadernejad, S., Dolatabadi, A., 2015, Coatings, 5(4), pp. 576-645.

[12] Dalir, E., Dolatabadi, A., Mostaghimi, J., 2019, Journal of Thermal Spray Technology, 28(6), pp. 1105-1125.

[13] Neural Networks and Deep Learning, http://neuralnetworksanddeeplearning.com/.

[14] Jadidi, M., Sreekumar, V., Dolatabadi, A., 2019, Journal of Visualization, 22(2), pp. 259271.

[15] Sovani, S., Sojka, P., Lefebvre, A., 2001, Progress in Energy and Combustion Science, 27(4), pp. 483-521.

[16] Lin, K.C., Kennedy, P.J., Jackson, T.A., Jan. 14-17 2002, 40th AIAA Aerospace Sciences Meeting and Exhibit.

[17] Saleh, A., Amini, G., Dolatabadi, A., 2018, Atomization and Sprays, 28(2), pp. 91-110.

[18] Jadidi, M., Kostic, S., Zimmer, L., Dworkin, S.B., 2020, Energies, 13(18), p. 4787. 